\title{
Analyzing the Safety Impacts of Variable Speed Limit Control on Aggregated Driving Behavior Based on Traffic Big Data
}

\author{
Xu Qu $\mathbb{D}^{1,},{ }^{1,2}$ Mofeng Yang $\mathbb{D}^{3},{ }^{3}$ Junyi Ji $\mathbb{D}^{1},{ }^{1}$ Linheng Li $\mathbb{D}^{1,2}$ and Bin Ran $\mathbb{D}^{1,2,4}$ \\ ${ }^{1}$ School of Transportation, Southeast University, Nanjing, China \\ ${ }^{2}$ Jiangsu Province Collaborative Innovation Center of Modern Urban Traffic Technologies, Southeast University Road \#2, \\ Nanjing 211189, China \\ ${ }^{3}$ Department of Civil and Environmental Engineering, University of Maryland-College Park, College Park, MD 20742, USA \\ ${ }^{4}$ Department of Civil and Environmental Engineering, University of Wisconsin-Madison, 1204 Engineering Hall, \\ 1415 Engineering Drive, Madison, WI 53706, USA
}

Correspondence should be addressed to Xu Qu; quxu@seu.edu.cn

Received 2 July 2020; Revised 9 August 2020; Accepted 19 March 2021; Published 26 March 2021

Academic Editor: Francesco Viti

Copyright (C) $2021 \mathrm{Xu}$ Qu et al. This is an open access article distributed under the Creative Commons Attribution License, which permits unrestricted use, distribution, and reproduction in any medium, provided the original work is properly cited.

Variable speed limit (VSL) control dynamically adjusts the displayed speed limit to harmonize traffic speed, prevent congestions, and reduce crash risks based on prevailing traffic stream and weather conditions. Previous research studies examine the impacts of VSL control on reducing corridor-level crash risks and improving bottleneck throughput. However, less attention focuses on utilizing real-world data to see how compliant the drivers are under different VSL values and how the aggregated driving behavior changes. This study aims to fill the gap. With the high-resolution lane-by-lane traffic big data collected from a European motorway, this study performs statistical analysis to measure the difference in driving behavior under different VSL values and analyze the safety impacts of VSL controls on aggregate driving behaviors (mean speed, average speed difference, and the percentage of small space headway). The data analytics show that VSL control can effectively decrease the mean speed, the speed difference, and the percentage of small space headways. The safety impacts of VSL control on aggregated driving behavior are also discussed. The aggregated driving behavior variables follow a trend of first decreasing and then increasing with the continuous decrease in VSL values, indicating that potential traffic safety benefits can be achieved by adopting suitable VSL values that match with prevailing traffic conditions.

\section{Introduction}

Variable speed limit (VSL) control dynamically adjusts the displayed speed limit on the variable message signs to harmonize traffic speed, prevent congestions, and reduce crash risks based on prevailing traffic stream and weather conditions, which is an essential control strategy for Active Traffic Management (ATM) system. Germany was among the earliest countries that implemented the VSL system in the mid-1960s [1]. Nowadays, VSL control has been widely adopted by countries including Germany, America, the Netherlands, United Kingdom, and China for real-time operation of motorway traffic to improve traffic safety and mitigate congestions [2].
To evaluate the safety benefits of the VSL control, many approaches have been taken. Questionnaire surveys are used to evaluate the safety benefits of VSL $[3,4]$. Their results showed that around $95 \%$ of drivers believe that VSL can effectively improve driving safety. The driving simulator is another way to examine the impact of VSL on driving behavior. A previous study shows that VSL can improve traffic safety by reducing speed differences between vehicles [5]. However, both questionnaire surveys and driving simulators are biased towards respondents' subjective factors, which might lead to the overestimation of VSL systems' safety benefits. Traffic simulation methods have been widely used to examine both the operation and safety benefits of different ATM control strategies [6-9]. To better reproduce the real- 
world effect of VSL control, different traffic simulation methods have also been applied to evaluated VSL benefits by simulating different driver groups' behavioral responses to the VSL [7-9]. Some researchers integrated a real-time crash potential prediction model that utilizes the output of the microscopic simulation [10-13]. They found that VSL could achieve safety benefits, especially in reducing the risk of rearend collisions. The simulation-based studies are able to quantitatively evaluate VSL safety impacts; however, the driving behavior is heterogeneous between different driver groups. For instance, aggressive drivers tend to drive with a higher desired speed compared to conservative drivers [14]. The microscopic simulation models simply apply the compliance rate to the driver group and universally set the compliant drivers' desired speed equalling to VSL values, which had not been proven correct by any empirical observations.

Empirical data, e.g., the loop detector data, has been widely used to analyze different aspects of motorway traffic $[15,16]$. With the support of these high-resolution data, driving behaviors under VSL control have also been well studied [17-23]. The consensus emerging from these studies is that VSL control can improve safety by decreasing the mean speed, the speed difference, and the percentage of small-time headway and increase the mean time headway. Studies using empirical traffic data as inputs can objectively analyze the impacts of VSL on driving behaviors and derive credible conclusions. However, with the constraint of predefined VSL control strategies, the majority of these studies only have access to data from a limited range of traffic conditions and limited VSL values. Therefore, most of these studies merely performed qualitative comparisons between driving behavior parameters with and without VSL controls, while missing the quantitative examination of the relationship between traffic variables and VSL values. In the realworld implementation, VSL values vary in accordance with the prevailing traffic conditions and weather. Therefore, such studies were not able to evaluate the potential outcomes of each VSL value with a limited amount of data. In the meantime, few studies have controlled traffic states when examining VSL impacts; therefore, it is difficult to determine whether the obtained results are caused by VSL control or by the difference in traffic states within the collected empirical data.

According to the literature, the understanding of VSL control's mechanisms leading to efficiency and safety is still not fully explored and understood by researchers. Efforts devoted to investigating the impacts of VSL on driving behaviors have been insufficient, especially for quantitative research studies on VSL. To the best knowledge of the authors, few have examined the impact of VSL control on the aggregated driving behaviors with different VSL values [24].

To fill the gap, this study utilized high-resolution laneby-lane traffic big data from a European motorway under different VSL values. Then a broad range of traffic states, i.e., traffic density, is categorized and investigated under various VSL values. With controlling the traffic state, the relationships between aggregated driving behaviors and VSL values within different traffic density intervals have been quantified. Based on the quantitative analysis results, this study measures the difference in driving behavior under different VSL values and discusses the safety impacts of VSL control on aggregated driving behaviors and potential improvement on the motorway rear-end collisions. The results of this study revealed the mechanism leading to the safety benefits of VSL control and provided more realistic assumptions for modeling traffic flow operations under VSL control. Furthermore, we also discussed the invaluable insights into developing effective VSL control strategies to improve traffic safety.

\section{Analyzing Aggregated Driving Behavior with Traffic Big Data}

2.1. Traffic Big Data. The high-resolution lane-by-lane traffic big data is collected from a two-direction European motorway segment with three lanes in each direction. Inductive loop detectors are placed every 500-meter on the motorway collecting traffic stream speed, headway, vehicle length, and other traffic flow characteristics. The motorway segment experiences a wide variety of traffic conditions (including recurrent and nonrecurrent congestions). A VSL control system is deployed along the motorway with the control objective of alleviating roadway congestions and improving traffic safety. A wide variety of speed limits including $50 \mathrm{~km} /$ $\mathrm{h}, 60 \mathrm{~km} / \mathrm{h}, 80 \mathrm{~km} / \mathrm{h}, 100 \mathrm{~km} / \mathrm{h}$, and $120 \mathrm{~km} / \mathrm{h}$ are implemented in the system.

This study uses traffic flow data and corresponding VSL control speeds data from 7:00 am to 6:00 pm for two weeks at a no weaving area site. The data only in good weather condition (no rain or fog) and visibility conditions (at daytime) is selected to avoid external interference. Missing and wrong data was removed. Besides, in order to eliminate drivers' adjustments immediately after the VSL control is triggered, the traffic flow data within 3 minutes after the implementation of VSL control is removed. Finally, the dataset including 4266 minutes data records with 355,599 vehicles was established, including 128,998 vehicles, 136,301 vehicles, and 90,300 vehicles on the left lane, middle lane, and right lane, respectively.

2.2. Traffic States. The investigation of the VSL impacts on driving behavior must control the traffic states. Under various traffic states, the driving behavior will be differently affected by the VSL control system. Therefore, the classification of the samples according to traffic states is indeed critical to this study. In this study, traffic density is chosen as a critical indicator to measure traffic congestion. The samples are further classified into different traffic density intervals to evaluate the impact of VSL control under respective traffic states. And the samples in the same density interval with and without VSL controls are also compared to investigate the effect of VSL control on driving behavior.

Furthermore, traffic data needs to be aggregated for the density parameter of every sample. The traffic volume, the mean speed, and the density of every sample are calculated 
using one minute as the statistical time interval. The traffic flow data within different density intervals under different VSL control speeds on different lanes are classified by five vehicles per kilometer per lane density intervals. Meanwhile, to ensure there are enough samples to perform statistical analysis, the density intervals under each VSL value whose sample size is too low should be ignored. Through trial and error, fifteen is selected as the lower limit of the sample size. The number of samples within different density intervals under different speed limits on different lanes is listed in Table 1 ("-" indicates that the number of samples is less than 15 within the associated density interval). Samples with high traffic density (larger than $30 \mathrm{veh} / \mathrm{km}$ ) are removed because, under high traffic density, the reduction of traffic speed is mainly caused by traffic congestions and stop-andgo waves, thus making it hard to quantify the actual impact of VSL on the aggregated driving behavior.

2.3. Aggregated Driving Behavior Variables. In this study, we defined three variables to represent the aggregated driving behavior: mean speed, average speed difference, and the percentage of small space headway. These three variables are calculated using the traffic big data and are further used to measure the safety impact of the VSL control. Definition and calculation for these three aggregated driving behavior variables are shown below.

2.3.1. Mean Speed. The two most frequently used mean speeds in traffic studies are the time mean speed and the space mean speed. This study selected the space mean speed as one of the analysis variables. It is the arithmetic mean speed value of vehicles within a certain section at a moment, which equals the harmonic mean of spot speed observed.

$$
\bar{v}_{s}=\frac{n}{\sum_{m=1}^{n} 1 / v_{m}},
$$

where $v_{m}$ represents the instantaneous velocities of the vehicle $m$; $n$ represents the number of vehicles passing the roadway segment.

2.3.2. Average Speed Difference (ASD). The average speed difference of two neighboring vehicles was selected to measure the speed difference. It is the mean value of speed difference between the neighboring vehicles passing a given point in a specified time interval [25].

$$
\mathrm{ASD}=\overline{\Delta v}=\frac{\sum_{i=1}^{n-1}\left|v_{i}-v_{i+1}\right|}{n-1},
$$

where $v_{i}$ represents the speed of the $i^{t h}$ vehicle passing the fixed point; $n$ represents the number of vehicles passing a given point.

2.3.3. The Percentage of Small Space Headway. Space headway is a measurement of the minimum possible distance between vehicles without a reduction in the speed of the following vehicles. A small disturbance might still lead to instability conditions on motorways when a platoon of vehicles is operating with small headways [26]. Therefore, the distribution of headway, particularly the percentage of small headways, has some influence on the stability and safety of traffic stream operations. The percentage of less than 100 meters of space headway was used as one of the analysis variables.

\section{Results}

The impact of VSL control on aggregated driving behavior is measured based on the mean speed, the variation of speeds, and headways in this study as introduced before.

3.1. VSL Impacts on Mean Speed. In order to compare the effect of different VSL values on the mean speed, the arithmetic average of all the sample's mean speed within different density intervals is calculated under different speed limits on different lanes. The results are summarized in Table 2. The two-sample Student's $t$-test is used to compare the mean speeds' difference with and without VSL controls, and the associated $p$ values for $t$-tests are also provided in parenthesis to infer the impact significance of the VSL control. The null hypothesis $\left(\mathrm{H}_{0}\right)$ is that the index is the same for the conditions with and without control. If the $t$-test value is less than 0.05 , we accept the hypothesis, if not, we reject it. The t-statistics is calculated using the formula shown below:

$$
t=\frac{\bar{x}_{1}-\bar{x}_{2}}{\sqrt{s_{1}^{2} / n_{1}+s_{2}^{2} / n_{2}}}
$$

where $\bar{x}_{1}, \bar{x}_{2}$ respectively, represent sample means of the two groups, $s_{1}^{2}, s_{2}^{2}$ represent the sample variances, and $n_{1}, n_{2}$ represent the sample sizes.

The results in Table 2 reveal that the mean speeds are lower under VSL controls regardless of the traffic density interval or lane locations. Under noncongested traffic density conditions (5-20 veh/km), the speed limit of $120 \mathrm{~km} /$ $\mathrm{h}$ only provides a marginal reduction in mean speeds on the left lane and the middle lane, yet the $t$-test results show that these speed differences are still statistically significant; meanwhile, other lower VSL speed values (lower than $120 \mathrm{~km} / \mathrm{h}$ ) are significantly reducing the mean speeds, and the $t$-test results for these speeds are also statistically significant. Under congested conditions (25-30 veh/ $\mathrm{km}$ on the left and the middle lane, $20-25 \mathrm{veh} / \mathrm{km}$ on the right lane), the $t$-test results reveal that the speed limit of $120 \mathrm{~km} / \mathrm{h}$ is not significantly changing the mean traffic stream speed when compared to conditions without VSL control; for other lower VSL values, obvious mean speed reductions are achieved, and these mean speed differences are all statistically significant.

To evaluate the impact of different VSL values for each lane under the same traffic state, enough samples need to be obtained under each VSL value for a given traffic density interval. Given the sample size information shown in $\mathrm{Ta}-$ ble 1 , only the traffic density interval $20-25 \mathrm{veh} / \mathrm{km}$ has enough sample size under all VSL values. Therefore, 
TABLE 1: Number of samples within different density intervals under different speed limits (“—” means the sample size is less than 15).

\begin{tabular}{|c|c|c|c|c|c|c|c|}
\hline \multirow{2}{*}{ Lane } & \multirow{2}{*}{ Density $(\mathrm{veh} / \mathrm{km})$} & \multicolumn{6}{|c|}{ Number of samples under different speed limits $(\mathrm{km} / \mathrm{h})$} \\
\hline & & No VSL & $120 \mathrm{~km} / \mathrm{h}$ & $100 \mathrm{~km} / \mathrm{h}$ & $80 \mathrm{~km} / \mathrm{h}$ & $60 \mathrm{~km} / \mathrm{h}$ & $50 \mathrm{~km} / \mathrm{h}$ \\
\hline \multirow{6}{*}{ Left lane } & $0-5$ & 217 & 28 & 18 & - & - & - \\
\hline & $5-10$ & 621 & 147 & 83 & - & - & - \\
\hline & $10-15$ & 496 & 178 & 103 & 18 & - & - \\
\hline & $15-20$ & 317 & 395 & 94 & 30 & 16 & - \\
\hline & $20-25$ & 221 & 687 & 54 & 48 & 45 & 15 \\
\hline & $25-30$ & 101 & 315 & 19 & - & - & - \\
\hline \multirow{5}{*}{ Middle lane } & $5-10$ & 97 & 21 & - & - & - & - \\
\hline & $10-15$ & 476 & 194 & 79 & - & - & - \\
\hline & $15-20$ & 1020 & 804 & 219 & 60 & 15 & - \\
\hline & $20-25$ & 345 & 637 & 66 & 18 & 30 & 16 \\
\hline & $25-30$ & 50 & 119 & - & - & - & - \\
\hline \multirow{4}{*}{ Right lane } & $5-10$ & 325 & 175 & 31 & - & - & - \\
\hline & $10-15$ & 1220 & 875 & 242 & 49 & - & - \\
\hline & $15-20$ & 412 & 626 & 99 & 31 & 38 & 15 \\
\hline & $20-25$ & 37 & 91 & - & - & - & - \\
\hline
\end{tabular}

TABLE 2: The arithmetic average and $t$-test results of mean speed within different density intervals under different speed limits on different lanes (“-” means the sample size is less than 15).

\begin{tabular}{|c|c|c|c|c|c|c|c|}
\hline \multirow{2}{*}{ Lane } & \multirow{2}{*}{ Density (veh/km) } & \multicolumn{6}{|c|}{ The arithmetic average of mean speed under different speed limits $(\mathrm{km} / \mathrm{h})$} \\
\hline & & No VSL & $120 \mathrm{~km} / \mathrm{h}$ & $100 \mathrm{~km} / \mathrm{h}$ & $80 \mathrm{~km} / \mathrm{h}$ & $60 \mathrm{~km} / \mathrm{h}$ & $50 \mathrm{~km} / \mathrm{h}$ \\
\hline \multirow{6}{*}{ Left lane } & $0-5$ & 129.6 & $125.7(0.00)$ & $111.4(0.00)$ & - & - & - \\
\hline & $5-10$ & 126.1 & $124.8(0.00)$ & $109.9(0.00)$ & - & - & - \\
\hline & $10-15$ & 122.5 & $121.4(0.00)$ & $106.3(0.00)$ & $98.0(0.00)$ & - & - \\
\hline & $15-20$ & 117.6 & $113.2(0.00)$ & $102.1(0.00)$ & $91.3(0.00)$ & $91.4(0.00)$ & - \\
\hline & $20-25$ & 111.6 & $106.3(0.00)$ & $96.4(0.00)$ & $86.5(0.00)$ & $86.4(0.00)$ & $90.6(0.00)$ \\
\hline & $25-30$ & 100.6 & $99.6(\mathbf{0 . 5 6})$ & $85.2(0.00)$ & - & - & - \\
\hline \multirow{5}{*}{ Middle lane } & $5-10$ & 116.9 & $110.2(0.00)$ & - & - & - & - \\
\hline & $10-15$ & 115.1 & $111.3(0.00)$ & $98.5(0.00)$ & - & - & - \\
\hline & $15-20$ & 111.8 & $104.3(0.00)$ & $96.7(0.00)$ & $84.4(0.00)$ & $82.0(0.00)$ & - \\
\hline & $20-25$ & 105.1 & $97.8(0.00)$ & $91.8(0.00)$ & $84.0(0.00)$ & $80.6(0.00)$ & $87.0(0.00)$ \\
\hline & $25-30$ & 85.7 & $88.9(\mathbf{0 . 1 8})$ & - & - & - & - \\
\hline \multirow{4}{*}{ Right lane } & $5-10$ & 98.8 & $92.5(0.00)$ & $88.8(0.00)$ & - & - & - \\
\hline & $10-15$ & 97.6 & $90.7(0.00)$ & $88.6(0.00)$ & $78.4(0.00)$ & - & - \\
\hline & $15-20$ & 92.6 & $85.9(0.00)$ & $86.0(0.00)$ & $78.1(0.00)$ & $72.7(0.00)$ & $77.0(0.00)$ \\
\hline & $20-25$ & 80.4 & $81.3(\mathbf{0 . 6 7})$ & - & - & - & - \\
\hline
\end{tabular}

20-25 veh/km was selected for the left lane and middle lane, and $15-20 \mathrm{veh} / \mathrm{km}$ was selected for the right lane to demonstrate the change trends in the mean speed under different speed limits. As shown in Figure 1, it can be observed that, after the $60 \mathrm{~km} / \mathrm{h}$ speed limit, the mean speeds increase with the speed limits reduction. The reason might be that some drivers might challenge the rationality of VSL controls when a low-speed limit is implemented, whereby the reduced VSL compliance rate leads to the phenomenon of higher mean speeds under lower VSL values.

3.2. VSL Impacts on ASD. In order to compare the effects of different speed limits on the ASD, the arithmetic average of all the sample's ASD within different density intervals is calculated under different speed limits on different lanes. Table 3 summarizes the results. Again, Student's $t$-test is used to compare the ASDs' difference between VSL control groups and No VSL groups, and the associated $p$ values are also provided in parenthesis.

It can be observed from Table 3 that, for all the lanes within the same density intervals, the ASDs are always lower under VSL controls. In addition, the $t$-test results show that the difference in ASDs is statistically significant for the majority of conditions except for speed limits of $120 \mathrm{~km} / \mathrm{h}$ on the left and middle lane within low-density intervals and speed limits of $60 \mathrm{~km} / \mathrm{h}$ on the left lane within the $15-20 \mathrm{veh} /$ $\mathrm{km}$ density interval.

Figure 2 describes the change trends in ASD under different speed limits on the left and middle lane within the $20-25 \mathrm{veh} / \mathrm{km}$ density interval and on the right lane within the $15-20 \mathrm{veh} / \mathrm{km}$ density interval. It is clear that ASDs first decrease and then increase with the reduction in speed limits. A possible explanation for this phenomenon is that when VSL speed values are low, those aggressive drivers might choose to retain their speeds or only adopt a small 


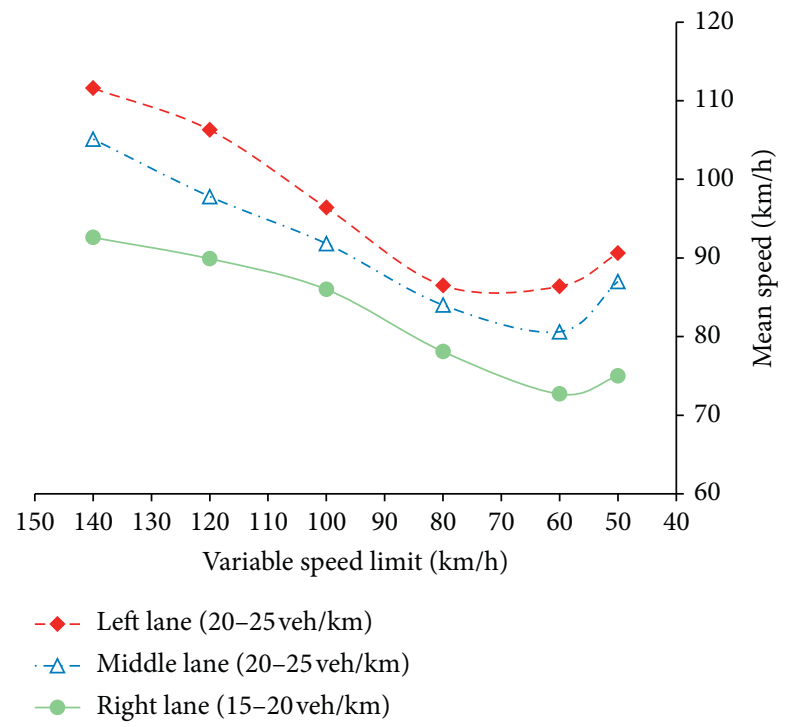

Figure 1: Change trends in mean speed under different speed limits.

TABLE 3: The arithmetic average and $t$-test results of ASD within different density intervals under different speed limits on different lanes (“-” means the sample size is less than 15).

\begin{tabular}{|c|c|c|c|c|c|c|c|}
\hline \multirow{2}{*}{ Lane } & \multirow{2}{*}{ Density (veh/km) } & \multicolumn{6}{|c|}{ The arithmetic average of ASD under different speed limits $(\mathrm{km} / \mathrm{h})$} \\
\hline & & No VSL & $120 \mathrm{~km} / \mathrm{h}$ & $100 \mathrm{~km} / \mathrm{h}$ & $80 \mathrm{~km} / \mathrm{h}$ & $60 \mathrm{~km} / \mathrm{h}$ & $50 \mathrm{~km} / \mathrm{h}$ \\
\hline \multirow{6}{*}{ Left lane } & $0-5$ & 8.89 & $7.01(0.01)$ & $6.85(0.02)$ & - & - & - \\
\hline & $5-10$ & 6.54 & $6.23(0.08)$ & $5.88(0.00)$ & - & - & - \\
\hline & $10-15$ & 5.13 & $4.97(\mathbf{0 . 1 4})$ & $4.53(0.00)$ & $4.41(0.03)$ & - & - \\
\hline & $15-20$ & 4.20 & $3.90(0.00)$ & $3.53(0.00)$ & $3.62(0.00)$ & $4.11(\mathbf{0 . 7 3})$ & - \\
\hline & $20-25$ & 3.61 & $3.24(0.00)$ & $3.12(0.00)$ & $2.95(0.00)$ & $3.15(0.00)$ & $3.19(0.03)$ \\
\hline & $25-30$ & 3.10 & $2.87(0.00)$ & $2.90(0.03)$ & - & - & - \\
\hline \multirow{5}{*}{ Middle lane } & $5-10$ & 7.80 & $7.78(0.97)$ & - & - & - & - \\
\hline & $10-15$ & 6.35 & $5.92(0.00)$ & $4.55(0.00)$ & - & - & - \\
\hline & $15-20$ & 5.37 & $4.69(0.00)$ & $3.82(0.00)$ & $3.44(0.00)$ & $5.03(0.00)$ & - \\
\hline & $20-25$ & 4.43 & $3.62(0.00)$ & $3.19(0.00)$ & $3.38(0.00)$ & $3.26(0.00)$ & $3.67(0.01)$ \\
\hline & $25-30$ & 3.32 & $2.86(0.00)$ & - & - & - & - \\
\hline \multirow{4}{*}{ Right lane } & $5-10$ & 10.19 & $8.75(0.01)$ & $5.63(0.00)$ & - & - & - \\
\hline & $10-15$ & 7.91 & $6.33(0.00)$ & $4.57(0.00)$ & $3.47(0.00)$ & - & - \\
\hline & $15-20$ & 6.02 & $4.48(0.00)$ & $3.55(0.00)$ & $3.10(0.00)$ & $3.64(0.00)$ & $4.15(0.01)$ \\
\hline & $20-25$ & 4.21 & $3.58(0.01)$ & - & - & - & - \\
\hline
\end{tabular}

speed reduction, while those conservative drivers will follow the speed limits and apply a large speed reduction. Such behavioral differences lead to a more discrete distribution for drivers' desired speeds, whereby resulting in higher ASD values in the traffic flow.

3.3. VSL Impacts on Space Headway. In order to quantify the effects of different VSL values on the space headway, the percentage of small headways within different density intervals is calculated under different speed limits on different lanes. Table 4 summarizes the percentage of vehicles with smaller than $100 \mathrm{~m}$ space headway under all combinations of density intervals and speed limits on the left, the middle, and the right lane ("- indicates that less than 300 vehicles are observed within the associated density interval).

It can be observed from the above table that the majority of percentage (of less than $100 \mathrm{~m}$ headway) results on the left and middle lane are smaller for VSL control groups when compared to No VSL groups. However, most percentage results on the right lane with VSL control are similar to the results of No VSL controls except for the speed limit of $100 \mathrm{~km} / \mathrm{h}$. The speed limit of $60 \mathrm{~km} / \mathrm{h}$ on the right lane increases the percentage of less than $100 \mathrm{~m}$ headway. In general, the percentages of less than $100 \mathrm{~m}$ headway first decrease and then increase for all lane locations within the 


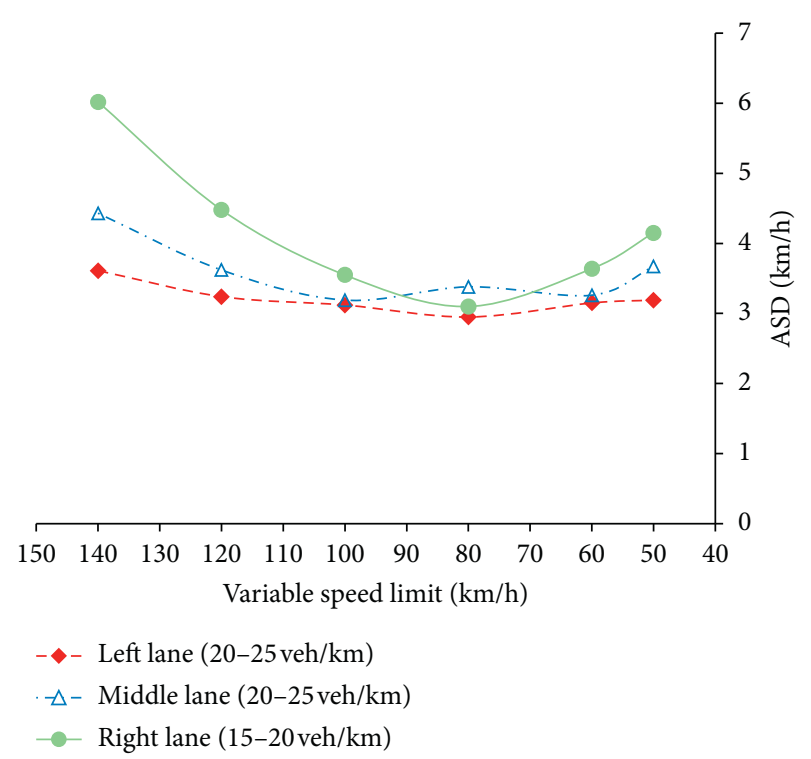

Figure 2: Change trends in ASD under different speed limits.

same density intervals with the lowest rate achieved at the speed limit of $100 \mathrm{~km} / \mathrm{h}$. Further reduction in speed limits beyond $100 \mathrm{~km} / \mathrm{h}$ will not further reduce the percentage of small-time headways.

3.4. VSL Impacts on Motorway Rear-End Collisions. This section focuses on understanding the impacts of VSL on rear-end collisions because rear-end collision is the most frequent accident type on the motorway. Although some rear-end collisions have resulted from inappropriate driver behaviors, vehicle failure, and so on, most collisions are closely related to the vehicle speed, the car following distance, and the speed difference between consecutive vehicles.

It is assumed that two vehicles are running in the same lane on a motorway. As shown in Figure 3, the leading vehicle is running at the speed of $V_{1}$, and the following one at the speed of $V_{2}$ with the distance of $d$ from the former. If an emergency happened ahead forcing the leading vehicle driver to brake immediately and adjust its speed to $V_{0}$ at the deceleration rate of $a$, the following driver also needs to brake to avoid a collision and needs to adjust its speed to at most $V_{0}$. The following vehicle's deceleration is assumed to be the same as the leading vehicle, and the drivers' reaction times are ignored.

The distance of the leading vehicle traveled:

$$
S_{1}=\frac{V_{0}^{2}-V_{1}^{2}}{2 a} \text {. }
$$

The distance of the following vehicle traveled:

$$
S_{2}=\frac{V_{0}^{2}-V_{2}^{2}}{2 a} \text {. }
$$

For avoiding a rear-end collision between the consecutive vehicles, the following condition shall be met:

$$
d>S_{2}-S_{1}=\frac{V_{1}^{2}-V_{2}^{2}}{2 a}=\frac{\left(V_{1}-V_{2}\right)\left(V_{1}+V_{2}\right)}{2 a} .
$$

A rear-end collision would not happen if the speed of the following vehicle $V_{2}$ is smaller than that of the leading one $V_{l}$. However, when the speed relationship is reversed, whether the collision would occur will depend on the distance $d$, the speed difference between the consecutive two vehicles $V_{2}-V_{1}$, the sum of the speed of two vehicles $V_{2}+V_{1}$, and the deceleration rate $a$. The results in the previous section showed that suitable VSL control can decrease the mean speed, the ASD, and the percentage of relatively small space headway (which means most drivers prefer to keep a large space headway). As a result, under reasonable VSL controls, the speed difference between the two consecutive vehicles $V_{2}-V_{1}$ and the sum of the two vehicles' speeds both become smaller in the right part of (5). Meanwhile, the possibility of space headway $d$ in the left part of (5) is also small. Therefore, it can be inferred that appropriate VSL controls can decrease the risk of rear-end collisions.

3.5. Driver's Compliance under VSL. This section focuses on understanding the driver's compliance under VSL because the driver's compliance determines the efficiency of the VSL. Based on our analysis, it can be observed that a lower VSL value does not guarantee a higher impact on driving behavior. For instance, under the low VSL values, the mean speed, average speed difference, and the percentage of small headways are greater than the same measurements under high VSL values. The reason for this phenomenon is the change in the driver's compliance with the variable speed limit control [8].

Whether or not the driver complies with the VSL is a process of measuring the greater of the benefits and the losses. From the driver's perspective, the benefits that may be gained from complying with the VSL include improved driving safety and no risk of penalties for speeding; the corresponding losses include reduced vehicle speed and increased travel time. On the contrary, if drivers fail to comply with the VSL, the possible benefits are efficiency benefits from higher driving speeds, etc.; the corresponding losses include reduced driving safety and the risk of penalties for speeding. Only when the driver judges that the benefits of the VSL are greater than the losses, they will choose to comply. When the VSL value is too low, that is, when the speed limit is significantly different from the driver's expected speed, the driver may question the rationality of the VSL. Under this situation, the driver's awareness of expecting to reach the destination as soon as possible defeats the awareness of complying with VSL limits to obtain safety benefits. At this time the driver will choose not to comply with the VSL.

Although the driver will choose not to follow the low VSL values, this does not mean that the driving behavior under low VSL values is the same as that without VSL. Our analysis finds that when the VSL values are too low, although no driver will choose a speed slower than the variable speed limit value, the mean speed is still significantly lower than the uncontrolled state. For example, under $60 \mathrm{~km} / \mathrm{h}$ VSL, the speed of almost all vehicles is higher than $60 \mathrm{~km} / \mathrm{h}$, and the 
TABLE 4: Percentages of smaller than 100 m headway within different density intervals under different speed limits (“-” means less than 300 vehicles are observed).

\begin{tabular}{|c|c|c|c|c|c|c|c|}
\hline \multirow{2}{*}{ Lane } & \multirow{2}{*}{ Density (veh/km) } & \multicolumn{6}{|c|}{ Percentage of less than $100-\mathrm{m}$ headway under different speed limits (\%) } \\
\hline & & No VSL (\%) & $120 \mathrm{~km} / \mathrm{h}(\%)$ & $100 \mathrm{~km} / \mathrm{h}$ & $80 \mathrm{~km} / \mathrm{h}$ & $60 \mathrm{~km} / \mathrm{h}$ & $50 \mathrm{~km} / \mathrm{h}$ \\
\hline \multirow{5}{*}{ Left lane } & $5-10$ & 23 & 21 & $15 \%$ & - & - & - \\
\hline & $10-15$ & 32 & 28 & $19 \%$ & $17 \%$ & - & - \\
\hline & $15-20$ & 35 & 26 & $15 \%$ & $18 \%$ & $14 \%$ & - \\
\hline & $20-25$ & 38 & 27 & $18 \%$ & $23 \%$ & $28 \%$ & $24 \%$ \\
\hline & $25-30$ & 42 & 33 & $33 \%$ & $38 \%$ & $42 \%$ & - \\
\hline \multirow{5}{*}{ Middle lane } & $5-10$ & 9 & 6 & - & - & - & - \\
\hline & $10-15$ & 15 & 11 & $8 \%$ & - & - & - \\
\hline & $15-20$ & 22 & 17 & $13 \%$ & $17 \%$ & - & - \\
\hline & $20-25$ & 28 & 22 & $19 \%$ & $23 \%$ & $35 \%$ & $21 \%$ \\
\hline & $25-30$ & 34 & 30 & $29 \%$ & - & - & - \\
\hline \multirow{4}{*}{ Right lane } & $5-10$ & 4 & 4 & - & - & - & - \\
\hline & $10-15$ & 9 & 9 & $8 \%$ & $9 \%$ & - & - \\
\hline & $15-20$ & 16 & 18 & $12 \%$ & $14 \%$ & $22 \%$ & - \\
\hline & $20-25$ & 29 & 27 & - & - & - & - \\
\hline
\end{tabular}

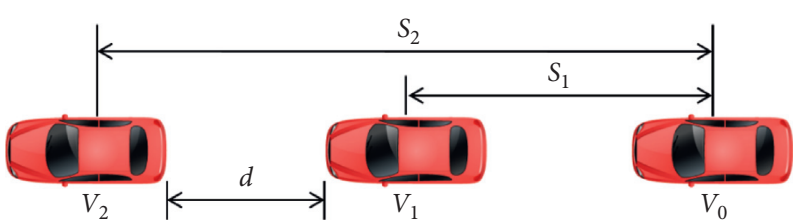

FIgURE 3: Schematic diagram of rear-end collisions.

mean speed is around $85 \mathrm{~km} / \mathrm{h}$, while the average speed in the uncontrolled state under the same density condition is around $110 \mathrm{~km} / \mathrm{h}$.

In the real world, each driver has an expected speed that considers safety and efficiency under any conditions. Drivers will drive as fast as possible based on ensuring driving safety and complying with traffic laws. The VSL is to change the driver's expected speed by changing the driver's safety expectations and the expectations of complying with traffic laws. Therefore, by popularizing the safety benefits of VSL and strengthening law enforcement for speeding violations, compliance with VSL can be effectively improved, thus improving the overall safety impacts of VSL.

\section{Conclusions and Discussion}

With the high-resolution lane-by-lane traffic big data collected from a European motorway, this study quantitatively measures the difference in driving behavior under different VSL values and analyzes the safety impacts of VSL controls on aggregate driving behaviors (described by mean speed, ASD, and the percentage of small space headway). Also, the potential improvement of VSL on rear-end collisions and the driver's compliance under VSL was discussed to prove that appropriate VSL controls could have provided additional traffic safety benefits. The main findings of this study are summarized as follows:
(1) Under medium and lower traffic density conditions, different VSL values can always reduce the mean traffic speeds. But the changes in the mean speed followed the first decreases and then increases trend with the reduction in speed limits. The result also indicated that, under low VSL values, drivers are more likely to challenge the rationality of the VSL system.

(2) Under similar traffic conditions, most VSL values reduced the speed differences between consecutive vehicles, thereby reducing the speed discretions in the traffic stream. Again, the first decreases and then increases trend with the reduction in speed limits in ASD showed that low VSL values may increase the ASD.

In general, VSL can reduce the mean speed, the speed difference, and the percentage of small headway. Reasonable VSL control strategies can effectively reduce the risk of rear-end collisions on the motorway. However, the first decreases and then increases trend with the reduction of speed limits illustrated that, under low VSL values, drivers are more likely to challenge the rationality of the VSL system. Therefore, the overly low VSL values cannot provide a higher safety benefit or even caused more traffic accidents. Thus, the implementation of the VSL control system should closely be related to the real-time traffic flow congestion level.

One limitation of this study is that it only considers the impact of VSL on the three aggregated driving behaviors under the same traffic density interval. On the other hand, VSL may have an impact on density under the same traffic demand. Future research is suggested to consider how VSL will impact the traffic density with the same traffic demand. In addition, the data used in this study does not include any lane-changing information. Since the lanechanging rate is another vital index for traffic stability and safety, the impact of VSL on the lane-changing rate is also 
encouraged to be analyzed with more detailed data collected.

\section{Data Availability}

The basic data used to support the findings of this study are available from the corresponding author upon request.

\section{Conflicts of Interest}

The authors declare that there are no conflicts of interest regarding the publication of this paper.

\section{Acknowledgments}

This research was supported by the National Key R\&D Program in China (Grant no. 2018YFB1600600), the MOE (Ministry of Education in China) Project of Humanities and Social Sciences (Project no. 20YJAZH083), and the National Natural Science Foundation of China (Grant no. 51878161).

\section{References}

[1] M. Papageorgiou, Applications of Automatic Control Concepts to Traffic Flow Modeling and Control, Springer, Berlin, Germany, 1983.

[2] B. Khondaker and L. Kattan, "Variable speed limit: an overview," Transportation Letters, vol. 7, no. 5, pp. 264-278, 2015.

[3] P. Rämä and J. Luoma, "Driver acceptance of weather-controlled road signs and displays," in Transportation Research Record: Journal of the Transportation Research Board, No.1573, pp. 72-75, Transportation Research Board of the National Academies, Washington, D.C., USA, 1997.

[4] P. Rämä, "Effects of weather-controlled variable speed limit and warning signs on driver behavior," in Transportation Research Record: Journal of the Transportation Research Board, No.1689, pp. 53-59, Transportation Research Board of the National Academies, Washington, D.C., USA, 1999.

[5] C. Lee and M. Abdel-Aty, "Testing effects of warning messages and variable speed limit on driver behavior using driving simulator," in Transportation Research Record: Journal of the Transportation Research Board, No.2069, pp. 55-64, Transportation Research Board of the National Academies, Washington, D.C., USA, 2008.

[6] M. Yang, Z. Li, Z. Ke, and M. Li, "A deep reinforcement learning-based ramp metering control framework for improving traffic operation at freeway weaving sections," in Proceedings of The 98th Annual Meeting of the Transportation Research Board, Washington, D.C., USA, January 2019.

[7] W. Zhou, M. Yang, M. Lee, and L. Zhang, "A Q-learning based coordinated variable speed limit and hard shoulder running control strategy to reduce travel time at freeway corridor," Transportation research record: Journal of the Transportation Research Board, vol. 2674, p. 915, 2020.

[8] X. Qu, L. Li, Z. Yi, P. Mao, and M. Yang, "Traffic flow modeling of freeway variable speed limit control based on the big data of driving behavior," Journal of Advanced Transportation, vol. 2020, Article ID 8859494, 11 pages, 2020.

[9] J. R. D. Frejo, I. Papamichail, M. Papageorgiou, and B. D. Schutter, "Macroscopic modeling of variable speed limits on freeways," Transportation Research Part C: Emerging Technologies, vol. 100, pp. 15-33, 2019.
[10] C. Lee, B. Hellinga, and F. Saccomanno, "Assessing safety benefits of variable speed limit," in Transportation Research Record: Journal of the Transportation Research Board, No.1897, pp. 183-190, Transportation Research Board of the National Academies, Washington, D.C., USA, 2004.

[11] M. Abdel-Aty, R. Cunningham, V. Gayah, and L. Hsia, "Dynamic variable speed limit strategies for real-time crash risk reduction on freeways," in Transportation Research Record: Journal of the Transportation Research Board, No.2078, pp. 108-116, Transportation Research Board of the National Academies, Washington, D.C., USA, 2008.

[12] E. Grumert, X. Ma, and A. Tapani, "Analysis of a cooperative variable speed limit system using microscopic traffic simulation," Transportation Research Part C: Emerging Technologies, vol. 52, pp. 173-186, 2015.

[13] X. Qu, W. Wang, W.-f. Wang, and P. Liu, "Real-time rear-end crash potential prediction on freeways," Journal of Central South University, vol. 24, no. 11, pp. 2664-2673, 2017.

[14] X. Qu, M. Yang, F. Yang, B. Ran, and L. Li, "An improved single-lane cellular automaton model considering driver's radical feature," Journal of Advanced Transportation, vol. 2018, no. 10, Article ID 3791820, 2018.

[15] C. Chen, K. Petty, A. Skabardonis, P. Varaiya, and Z. Jia, "Freeway performance measurement system: mining loop detector data," Transportation Research Record: Journal of the Transportation Research Board, vol. 1748, no. 1, pp. 96-102, 2001.

[16] M. Yang, J. Xie, P. Mao, C. Wang, and Z. Ye, "Application of the ARIMAX model on forecasting freeway traffic flow," in CICTP 2017: Transportation Reform and Change-Equity, Inclusiveness, Sharing, and Innovation, pp. 593-602, American Society of Civil Engineers, Reston, VA, USA, 2018.

[17] H. Zackor, "Self-sufficient control of speed on freeways," in Proceedings of International Symposium on Traffic Control Systems, pp. 226-249, University of California, Berkeley, CF, USA, December 1979.

[18] S. Smulders, "Control of freeway traffic flow by variable speed signs," Transportation Research Part B: Methodological, vol. 24, no. 2, pp. 111-132, 1990.

[19] E. Van den Hoogen and S. Smulders, Control by Variable Speed Signs: Results of the Dutch Experiment, IET, in Proceedings of Seventh International Conference on Road Traffic Monitoring and Control, pp. pp145-149, IET, London, UK, April 1994.

[20] T. Ha, J. Kang, and J. Park, The Effects of Automated Speed Enforcement Systems onTraffic-Flow Characteristics and Crashes in Korea, pp. 28-31, Institute of Transportation Engineers, Washington, DC, USA, 2003.

[21] P. Borrough, Variable Speed Limit Reduce Crashes Significantly in the UK, The Urban Transportation Monitor, 1997.

[22] Y. Pilli-Sivola, State of the Art in Finland Concerning RWIS and Variable Message Signs, Finnish National Road Administration, Helsinki, Finland, 2000.

[23] M. Papageorgiou, E. Kosmatopoulos, and I. Papamichail, "Effects of variable speed limit on motorway traffic flow," in Transportation Research Record: Journal of the Transportation Research Board, No. 2047, pp. 37-48, Transportation Research Board of the National Academies, Washington, D.C., USA, 2008.

[24] F. Soriguera, I. Martínez, M. Sala, and M. Menéndez, "Effects of low speed limits on freeway traffic flow," Transportation Research Part C: Emerging Technologies, vol. 77, pp. 257-274, 2017. 
[25] H. Wang, W. Wang, X. Chen, J. Chen, and J. Li, "Experimental features and characteristics of speed dispersion in urban freeway traffic," in Transportation Research Record: Journal of the Transportation Research Board, No.1999, pp. 150-160, Transportation Research Board of the National Academies, Washington, D.C., USA, 2007.

[26] U. Kohler, "Stability of vehicle platoons," Transportation and Traffic Theory, vol. 6, pp. 39-55, 1974. 